\title{
On the restraint mechanism between the earth and moon
}

\author{
Jian DING* \\ Retired, Integrated Electronic Systems Lab Co. Ltd., Jinan, China
}

\begin{abstract}
The moon always use the same side to face toward the earth, but there is a dead angle in the mainstream theory of explaining this phenomenon. That is, it cannot explain why the moon doesn't rotate around the axis which is a straight line to connect the mass centers of the earth and moon. Because the numerous meteorite impact craters on the lunar surface indicate that the moon is completely possible to obtain external momentums and rotate around this axis. This paper proposes a plain explanation, that is, the universal gravitation between the earth and moon as well as the earth's magnetic field have formed a trinity restraint mechanism on the moon. According to this explanation, the moon's rotation can be locked, and the mechanism of lunar libration has been revealed out, which can also confirm mutually with the natural phenomenon that the moon has sought a balance in the swing. In addition, with the help of all kinds of detection data from the Apollo moon landings and other circumlunar spacecraft, as well as the studies and analysis of lunar soil samples, the conclusion is that as far as a whole for the moon, it belongs to paramagnetic substances, and its relative permeability is between 1.008 and 1.03. Although the magnetic flux density of the earth on the lunar orbit has been dropped below $0.0008125 \mathrm{nT}$ or lower due to the impact of the solar wind, but it can be used as a reason to lock the moon without rotating around the axis which is a straight line to connect the mass centers of the earth and moon. If another main reason to cause the existence of this fact cannot be found, even if the magnetic flux density of the geomagnetism in lunar orbit is very small, it also should not be artificially ignored. In this regard, we can artificially change the intensity of the earth's magnetic field, and carefully observe the lunar libration and in the distance between the earth and the moon, to verify the arguments in this paper.
\end{abstract}

Key words: Moon; Earth; tidal locking; dipole magnetic field; solar wind; moonfall

PACS: $96.20 .-\mathrm{N} ;$ 96.20.Jz

* Corresponding author introduction: DING Jian, Male, Retired, metaphysics and physics. E-mail: jiandus@163.com 


\section{Introduction}

The moon is a celestial body closest to the earth, and also the only natural satellite of the earth. Its diameter is 3476 kilometers, the volume is equivalent to $1 / 49$ of the earth, but the mass is only $1 / 81$ of the Earth. Therefore, its average density is small, only equivalent to $3 / 5$ of the earth. The period of the moon's elliptical orbit around the earth is 27.3 days, the perigee is 363300 kilometers, the apogee is 405500 kilometers, and the average orbital radius is about 384,400 kilometers.

On the earth, we can only see the same side of the moon, while the other half of the moon is always facing away from the earth. Accordingly, the lunar side toward the earth is called the near side, and the other side is the far side. Conversely, if you standing on the near side of the moon to see the earth, in addition to the rotation, its position in the sky is always constant, just like we are standing in a room to watch a globe on the desk. Of course, you will not see the earth if standing on the far side of the moon.

\section{There is a dead angle in the theory of tidal locking}

The moon's rotation and orbital period are the same, so it is always with the same side toward the earth. This phenomenon is known as "synchronous rotation", and supplemented by the theory of "tidal locking" to explain ${ }^{[1]}$. Its meanings are briefly summarized below: The early moon orbit around the earth at the faster speed, it was not always with the same side toward the Earth. Due to the effect of tidal friction between the earth and the moon, part of the angular momentum of the earth's rotation was transferred to the moon, leading to the lunar orbital radius around the earth increased slightly. Of course, the speed of the earth's rotation has also been slowed down slightly. After long years, it has finally reached that the moon is always with the same side toward the Earth.

So-called the effect of tidal friction can only be transmitted through the universal gravitation between the earth and the moon. Because of this, in this theory of tidal locking, there must exist a dead angle. That is, it cannot explain why the moon does not rotate around the axis which is a straight line to connect the mass centers of the earth and moon. Why do we choose this straight line as an axis? Because the transmitted tidal friction force, which resulting torque relative to the axis should be zero. Even if it is not zero, locking the moon which has obtained external momentums and 
rotated around the axis, also takes a long time.

We all know that there are many of meteorite impact craters on the lunar surface, which indicate that the moon is completely possible to obtain external momentums and rotate around this axis. That is to say, when a random external momentum is obtained somewhere on the lunar surface, which can be decomposed into three vectors. The first vector acts on the moon's mass center, which can only cause the mass center to move. The second vector is perpendicular to the axis, thereby to form a torque relative to the axis. The third vector is parallel to the axis, and the results that it combine with the second vector can make us to see some information on the far side of the moon.

If there is the moon's rotation around the axis which is a straight line to connect the mass centers of the earth and moon, again then supplemented by the theory of tidal locking to explain, the mechanism that we do not see the far side of the moon may be available to attain perfection. In fact, we have only observed that the moon used this as the axis and sought a balance in the swing, and never reached the degree of rotation. This demonstrates that there are other acting forces between the earth and the moon. Therefore, it is necessary for us to rethink the restraint mechanism between the earth and moon.

\section{A plain explanation and the authoritative assertion}

We need to explain the question is why does the moon not rotate around the axis which is a straight line to connect the mass centers of the earth and moon? In this regard, this paper proposes a plain explanation, that is, the universal gravitation between the earth and moon as well as the earth's magnetic field to have formed a trinity restraint mechanism on the moon. Due to the characteristics of the moon itself, the location of the lunar mass center relative to its centroid has shifted about 2 kilometers toward the direction of the earth ${ }^{[2]}$, so the lunar mass center is not on the straight line connecting its two magnetic poles. It is the restraint mechanism of this triangle to cause that we can only see the same side of the moon.

In such a trinity restraint mechanism, once the moon obtaining a random external momentum, in addition to cause its mass center to be moved, it will also generate a torque relative to the axis which is a straight line to connect the mass centers of the earth and moon, and then rotate. At this point, it will inevitably cause the mechanism to generate an opposite force to correct the lunar mass center to 
be moved, while also to generate an opposite torque to stop the moon rotating around the axis. Of course, those moments in other directions are also stopped by this mechanism. Therefore, as a natural phenomenon, the moon has sought a balance in the swing. This is called the lunar libration, or Libration. As early as 17 th century, it has been observed by astronomers. As a result of the libration, the lunar surface that people can see on the earth can reach $59 \%$, and only the remaining $41 \%$ cannot be seen.

For this kind of the trinity restraint mechanism, there is a premise, that is, the moon must have a global dipole magnetic field like the earth. But regrettably, most of scientists having a consensus that the present-day moon has no global dipole magnetic field ${ }^{[3]}$, which was based on the resulting data obtained from Apollo moon landings and other circumlunar spacecraft in the last century. The detecting data obtained from the Apollo 15 and 16 subsatellites, Luna 10 and Explorer 35, and Lunar Prospector spacecraft, as well as other circumlunar spacecraft to show that the average magnetic flux density of the entire lunar surface was about $4 \mathrm{nT}$, and there were larger differences in different regions. Such as it was generally 0.75 - $6 \mathrm{nT}$ in the near side of the moon, and even more than 100 nT in individual regions. The Apollo moon program had carried out a total of 6 manned lunar landing, and there were 12 American astronauts landing on the moon. That was to say, if each astronaut to hold a compass which could be applied to 0.75 - $100 \mathrm{nT}$ magnetic flux density, the directions indicated by the compass were not the same, when they were in different regions of the moon.

\section{Estimating the strength of the earth's magnetic field in lunar orbit}

The earth has a global dipole magnetic field. Generally speaking, the magnetic flux density of the magnetic dipole at a certain point in space, is inversely proportional to the third power of the distance between this point and the center of the magnetic dipole ${ }^{[4]}$. The magnetic flux density on the earth's surface, is about 30,000 to 40,000 nT near the equator, $61000 \mathrm{nT}$ near the geomagnetic north pole, $68000 \mathrm{nT}$ near the geomagnetic south pole. It is well-known that the earth's average radius is 6371 kilometers, and the average distance between the earth and moon is about 384,400 kilometers. If the magnetic flux density of the earth's surface to adopt the average of $50000 \mathrm{nT}$, and let the coefficient $\mathrm{k}=1$, well, the earth as a magnetic dipole, whose magnetic flux density in lunar orbit can 
be roughly estimated. As follows:

$$
B_{1} \approx 50000 \times\left(\frac{6371}{384400}\right)^{3}=0.227637
$$

First of all, it is necessary to review the precision of the magnetometer carried by each lunar spacecraft. For example, it appeared difficult to competent because the precision of the magnetometer ${ }^{[5]}$ carried by the Lunar Prospector was only $0.2 \mathrm{nT}$. Moreover, due to the interaction between the solar wind and the earth's magnetic field, the result is that the earth's magnetic flux density is far less than $0.2 \mathrm{nT}$ in lunar orbit.

The solar wind is a kind of plasma, it also has a magnetic field. In the action against the earth's magnetic field, the solar wind bypasses the earth's magnetic field and continues to move forward. Thereupon, the earth's magnetic field is surrounded by the solar wind to form a region shaped like a comet, which is the earth's magnetosphere, and located at an altitude of 600 - 1000 kilometers above sea level. It is generally believed that at the altitude of 50,000 to 70,000 kilometers above sea level, the outer boundary of the magnetosphere has been formed, which is called the magnetopause. In there, the earth's magnetic field has been negligible, or may be considered to stop there.

But the fact was that based on the magnetic field observation data of the Apollo 15 subsatellite, the upper limit of the permanent dipole moment in lunar orbit was $1.3 \times 10^{18}$ (gauss-cubic centimeters) ${ }^{[6]}$. And based on the observation data of the earth's magnetic field in 1965, the geocentric magnetic dipole moment was about $8 \times 10^{25}$ (gauss-cubic centimeters) ${ }^{[7]}$. That is to say, as long as we take advantage of the ratio between the two, as well as the average value of the the magnetic flux density on the earth's surface is about 50,000 nT, so for the earth's magnetic field in the lunar orbit, its magnetic flux density can be roughly estimated. As follows:

$$
B_{2} \approx 50000 \times \frac{1.3 \times 10^{18}}{8 \times 10^{25}}=8.125 \times 10^{-4}
$$

\section{The main reason of the existence of things cannot be ignored}

In terms of the magnetic flux density of the geomagnetism in lunar orbit, although this estimated value is very small, but it should not be artificially ignored. Because it constitutes a basis to deny that the modern moon has no global dipole magnetic field. For the issues raised in this paper, that is why the moon not rotate around the axis which is a straight line to connect the mass centers of the earth and moon, if you cannot find another main reason to cause the existence of this fact, even if 
the magnetic flux density of the geomagnetism in lunar orbit is very small, it also should not be artificially ignored. Accordingly, the modern moon has no global dipole magnetic field, this authoritative assertion also must be re-examined.

We have known that according to the magnetic phenomena exhibited by substances under the action of external magnetic fields, usually can be divided into three categories of paramagnetic and diamagnetic and ferromagnetic. So, how should the moon behave under the action of external magnetic field?

In the Apollo moon landing project, there were a total of 12 NASA astronauts landed on the lunar surface, they also brought back 381.7 kilograms of lunar soil samples in addition to implement various types of exploration missions. The analysis of samples of lunar rocks showed that 3.1 billion years ago the moon had a stronger magnetic field ${ }^{[8]}$, and its intensity might be roughly the same as the magnetic field of our earth. If the moon once had a global dipole magnetic field, then there should have been an iron core ${ }^{[9]}$. But the modern moon had no global dipole magnetic field, and the various reliable evidences which led to this authoritative assertion have shown that the moon could not have such a core. The two arguments seemed to be opposites, but in essence they expressed a unified meaning, that was, in the moon must contain a fair amount of substances related to iron ${ }^{[10]}$.

In fact, it was Indeed so. The studies on the characteristics of remanent magnetization of lunar soil samples has shown that the irons in lunar soil have the superparamagnetic characteristic ${ }^{[11]}$. The implication is that there are pure irons which have no hysteresis, that is, whose remanence and coercive force are almost zero. In addition, the lunar soil also contains a large number of extremely rare metal elements on the earth, such as titanium, chromium and yttrium, and so on. These metals only at very high temperatures, about 2500 degrees Celsius or so, can be melted into the surrounding rock. These metals only at very high temperatures, about 2500 degrees Celsius or so, can be melted into the surrounding rock. Perhaps, at this moment, we should go to imagine broadly about that iron core of 3.1 billion years ago, how it was broken up the whole into parts, and turned into the superparamagnetic pure iron, and melted into the lunar soil.

More convincing measurement data was from the Explorer 35 spacecraft and the ALSEP automatic instrument station placed on the lunar surface, it showed that the moon as a whole was paramagnetic substances, and its relative permeability $\left(u_{r}=u / u_{0}\right)$ was between 1.008 and $1.03^{[6]}$. 


\section{Conclusion}

Rethinking the restraint mechanism between the earth and moon, this paper proposes a plain explanation which is the universal gravitation between the earth and moon as well as the earth's magnetic field to have formed a trinity restraint mechanism on the moon. This explanation does not exclude the theory of tidal locking, and can make up for the deficiency of the theory, that is, the moon can be locked and not rotate around the axis which is a straight line to connect the mass centers of the earth and moon. Furthermore, it can also explain the lunar libration, and confirm mutually with the natural phenomenon that the moon has sought a balance in the swing. But there must be a premise that the moon should have a global dipole magnetic field like the earth.

Apollo moon landings and other circumlunar spacecraft to detect as well as the studies of lunar soil samples have shown that there are a fair amount of pure irons with superparamagnetic characteristics in the moon. Therefore, as far as a whole for the moon, it belongs to paramagnetic substances, and its relative permeability is between 1.008 and 1.03. In view of this, if you want to assert that the modern moon has no global dipole magnetic field, there must be a prerequisite, that is, the effect of external constant magnetic field does not exist. That is to say, if there is a constant magnetic field in the lunar orbit, then the moon can certainly be magnetized, thus to form a global dipole magnetic field.

Of course, the constant magnetic field should be the earth's magnetic field. Although the magnetic flux density has been dropped below $8.125 \times 10^{-4} \mathrm{nT}$ or lower due to the impact of the solar wind, but it can be used as a reason to lock the moon without rotating around the axis which is a straight line to connect the mass centers of the earth and moon. If another main reason to cause the existence of this fact cannot be found, even if the magnetic flux density of the geomagnetism in lunar orbit is very small, it also should not be artificially ignored. In this regard, we can artificially change the intensity of the earth's magnetic field, and carefully observe the lunar libration and in the distance between the earth and the moon, to verify the arguments in this paper.

\section{References}

[1] C.D. Murray, S.F. Solar System Dynamics [M]. England: Cambridge University Press, 2000. 
[2] Kong X Y, Li J C, Guo J M, et al. Determination of gravitational constants for Earth and Moon and geocentric center and mass center of Moon [J]. Journal of Geodesy and Geodynamics, (in Chinese), 2006, 20(3): 109-114.

[3] Li Y Q, Liu J Z, Ouyang Z Y, et al. Lunar magnetism and lunar evolution [J]. Progress in Geophysics, (in Chinese), 2005, 20(4): 1003-1008.

[4] Ren L P, Zhao J S, Hou S X. The Distribution Model of Magnetic Dipole in Magnetic Field [J]. Hydrographic Surveying and Charting, (in Chinese), 2002, 22(2): 18-21.

[5] Xiao Z Y, Zeng Z X. New Progress of research on the Lunar magnetic field [J]. Progress in Geophysics, (in Chinese), 2010, 25(3): 804-808.

[6] Russell C T, Coleman P J, Schubert G Jr. Lunar magnetic field : permanent and induced dipole moments [J]. Science, 1974, 186: 825-826.

[7] Yang H Q. Thinking about the mechanism of geomagnetic polarity reversal [J]. Northwestern Geology, (in Chinese), 2002, 35(3): 126-130.

[8] Fuller M. Lunar magnetism--a retrospective view of the apollo sample magnetic studies [J]. Physics and Chemistry of the Earth, 1998, 23(7, 8): 725-735.

[9] Runcorn S K. The formation of the lunar core [J]. Geochimica et Cosmochimica Acta, 1996, 60: 1205-1208.

[10] Nagata T, Fisher R M, Schwerer F C. Lunar rock magnetism [J]. The Moon, 1972, 4: 170-196.

[11] Nagata T, Carleton B J. Natural remanent magnetization and viscous magnetization of apollo 11 lunar materials [J]. Journal of geomagnetism and geoelectricity, 1970, 22(4): 491-506. 